\title{
Quasi-Situ Characterization of Deformation in Low-Carbon Steel with Equiaxed and Lamellar Microstructure Treated by the Quenching and Partitioning Process
}

\author{
Pengfei Gao ${ }^{1,2} \cdot$ Weijian Chen ${ }^{1,2} \cdot$ Feng $\mathrm{Li}^{1,2} \cdot$ Beijia Ning ${ }^{1,2} \cdot$ Zhengzhi Zhao $^{1,2}$
}

Received: 26 March 2020 / Revised: 15 June 2020 / Accepted: 5 July 2020 / Published online: 15 September 2020

(c) The Chinese Society for Metals (CSM) and Springer-Verlag GmbH Germany, part of Springer Nature 2020

\begin{abstract}
The relationship between microstructure morphology and mechanical properties of the low-carbon steel (Fe-0.20C-2.59Mn$2.13 \mathrm{Si}$ ) treated by different intercritical annealed quenching and partitioning $(\mathrm{Q} \& \mathrm{P})$ processes was investigated through interrupted tensile tests plus quasi-situ electron backscatter diffraction measurements. Results show that size and distribution of retained austenite (RA) directly affect the sequence of deformation induced martensitic transformation. As strain increases, the equiaxed RA grains wrapped by ferrite transform first, followed by the equiaxed and film-like RA grains adjacent to martensite. Compared with traditional intercritical annealed Q\&P steel with equiaxed structure, the steel with quenching pretreatment contains uniform lamellar structure and the relatively film-like type of RA, leading to the higher yield strength, tensile strength, and elongation, as well as the steady increase in dislocation density upon straining.
\end{abstract}

Keywords Steel $\cdot$ Quenching and partitioning $\cdot$ Austenite $\cdot$ Martensitic transformation $\cdot$ Electron backscatter diffraction $($ EBSD) $\cdot$ Tensile test

\section{Introduction}

As a member of the third-generation advanced high strength steels (AHSS), the quenching and partitioning (Q\&P) steel has gained full attention in recent decades [1-4]. Proposed by Speer et al. [5], the Q\&P steel has a high elongation and an excellent strength-ductility balance. In particular, the intercritical annealed Q\&P steel, which obtains better comprehensive properties, has realized industrial production [6].

The intercritical annealed Q\&P heat treatment process prompts the low-carbon steel to form a multiphase

Available online at https://link.springer.com/journal/40195

Electronic supplementary material The online version of this article (https://doi.org/10.1007/s40195-020-01135-8) contains supplementary material, which is available to authorized users.

Zhengzhi Zhao

zhaozhzhi@ustb.edu.cn

1 Collaborative Innovation Center of Steel Technology, University of Science and Technology Beijing, Beijing 100083, China

2 Beijing Laboratory of Metallic Materials and Processing for Modern Transportation, Beijing 100083, China microstructure, including ferrite, martensite, and retained austenite (RA). Numerous studies have focused on the effect of phase volume fractions and grain size on mechanical properties and the relationship between the stability of RA and transformation-induced plasticity (TRIP) effect [7-9]. Knijf [10] demonstrated that the morphological characteristics (equiaxed or lamellar) of microstructure had a significant influence on the mechanical properties. Many studies have verified that the layered microstructure of steel helps obtain an excellent combination of strength and elongation [11-13]. This improvement in mechanical properties is attributed to continuous transformation of film-like RA observed by some researchers [14-16].

However, it is limited to study the microstructure evolution of materials during deformation under conventional quasi-static deformation. Using quasi-situ electron backscatter diffraction (EBSD) measurements [17-19] to study the difference between equiaxed and lamellar microstructure low-carbon steel treated by intercritical annealed quenching and partitioning process, we can clearly characterize the evolution of microstructure with strain and the reason why the lamellar structure steel has better properties at the same time. In the present work, two low-carbon steels treated by intercritical annealed Q\&P processes with different 
morphological characteristics of the microstructure were prepared. The equiaxed microstructure becomes lamellar by adding quenching pretreatment before the traditional Q\&P process. Mechanical properties and evolution of equiaxed and lamellar microstructure with strain were studied through interrupted tensile tests plus quasi-situ EBSD measurements.

\section{Experimental}

The investigated steel has a chemical composition of Fe-0.20C-2.59Mn-2.13Si (wt $\%$ ). The ingot was prepared by vacuum induction melting, reheated at $1200{ }^{\circ} \mathrm{C}$ for $2 \mathrm{~h}$ and then forged to $40 \mathrm{~mm}$. The forge piece was heated and hot-rolled to $2.8 \mathrm{~mm}$ and then cold rolled to a final thickness of $1.3 \mathrm{~mm}$.

Phase transformation temperatures, $A_{\mathrm{c} 3}, A_{\mathrm{c} 1}, M_{\mathrm{s}}$, and $M_{\mathrm{f}}$ (austenite transformation finishing temperature, austenite transformation starting temperature, martensite transformation starting temperature, and martensite transformation finishing temperature), were $890{ }^{\circ} \mathrm{C}, 710{ }^{\circ} \mathrm{C}, 365^{\circ} \mathrm{C}$, and $160{ }^{\circ} \mathrm{C}$, respectively, measured by the DIL805A thermal dilatometer. In order to obtain sufficient amount of stable RA, the intercritical annealed temperature of $800{ }^{\circ} \mathrm{C}$ was chosen, according to formula $\left(A_{\mathrm{c} 1}+A_{\mathrm{c} 3}\right) / 2$ [20]. After the cold-rolled sheet was heated at $800{ }^{\circ} \mathrm{C}$ for $120 \mathrm{~s}$, the $M_{\mathrm{s}}$, and $M_{\mathrm{f}}$ changed to $255^{\circ} \mathrm{C}$ and $70{ }^{\circ} \mathrm{C}$ due to the carbon enrichment in the austenite during the heating process, which was measured by thermal dilatometer. The quenching temperature of $150{ }^{\circ} \mathrm{C}$ was selected by using the constrained carbon equilibrium (CCE) model combined with the Koistinen-Marburger (K-M) equation [21, 22]. The Q\&P treatments were carried out using the CCT-AY-II heat treatment system for a thin steel sheet. As shown in Fig. 1, samples were heated to $800{ }^{\circ} \mathrm{C}$ at $10{ }^{\circ} \mathrm{C} / \mathrm{s}$ for $120 \mathrm{~s}$ for partial austenitization, followed by cooling at $45^{\circ} \mathrm{C} / \mathrm{s}$ to $150{ }^{\circ} \mathrm{C}$, then reheated and isothermally held at $420^{\circ} \mathrm{C}$ (partitioning

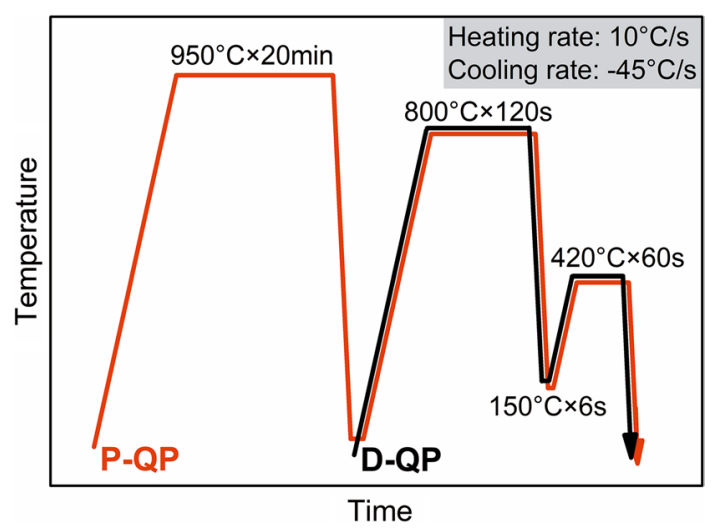

Fig. 1 Schematic diagrams of heat treatments temperatures) for $60 \mathrm{~s}$ before quenching $\left(-45^{\circ} \mathrm{C} / \mathrm{s}\right)$ to room temperature. A quenching pretreatment was implemented before Q\&P treatments to obtain the lamellar microstructure. Samples directly subjected to the Q\&P treatment were termed hereafter as D-QP, and samples pre-quenched before the $\mathrm{Q} \& \mathrm{P}$ procedure were termed hereafter as $\mathrm{P}-\mathrm{QP}$.

The microstructures were characterized by an FEI Quanta 450FEG field emission scanning electron microscope (SEM) operated at $20 \mathrm{kV}$. Metallographic samples were wire-cut, ground, polished, and etched with 3\% nital. EBSD was performed on the interrupted tensile sample with $20 \mathrm{kV}$ and a step size of $60 \mathrm{~nm}$ in PHI710 auger electron spectrometer with an EBSD detector. Detection surfaces of EBSD samples were prepared by electropolishing with a solution of $10 \%$ perchloric acid and 90\% ethanol (vol.\%) after precision grinding. The transmission electron microscopy (TEM) observation was performed in a JEM 2100 machine operated at $200 \mathrm{kV}$. Twin-jet polishing (5\% perchlorate alcohol at $-30{ }^{\circ} \mathrm{C}$, applied the potential of $50 \mathrm{~V}$ ) and ion beam thinning technique were implemented to prepare thin foil samples for TEM observation.

$\mathrm{X}$-ray diffraction (XRD) experiments were carried out by a Bruker D8 Advance diffractometer operated at $40 \mathrm{kV}$ and a current of $150 \mathrm{~mA}$ with $\mathrm{Cu} K_{\alpha}$ radiation. The austenite volume fraction was determined using Miller's empirical equation [23] based on XRD peak (Fig. S1) analysis as follows:

$V=0.7\left(I_{\gamma}^{220}+I_{\gamma}^{311}\right) /\left[I_{\alpha}^{211}+0.7\left(I_{\gamma}^{220}+I_{\gamma}^{311}\right)\right]$

where $I_{\alpha}^{\mathrm{hkl}}$ and $I_{\gamma}^{\mathrm{hkl}}$ are the integrated intensities of the $(h k l)$ peaks for body-centered-cubic (bcc) and face-centered-cubic (fcc) structure.

The tensile properties of samples with different morphological characteristics of microstructure were measured by the uniaxial tensile test with the standard specimen (according to the GB/T 228.1-2010 standard, gauge length: $25 \mathrm{~mm}$ ), of which the tensile direction is parallel to the rolling direction. The tests were performed with a quasi-static strain rate of about $3 \times 10^{-4} \mathrm{~s}^{-1}$ at room temperature, and an extensometer was used to measure the strain of gauge length of the samples.

Interrupted tensile tests were carried out to obtain the microstructure under different strains. The samples were tensioned with a quasi-static strain rate of about $3 \times 10^{-4} \mathrm{~s}^{-1}$ at room temperature. The tensile specimens were machined with a gauge length of $10 \mathrm{~mm}$ and a gauge width of $4 \mathrm{~mm}$ from the heat-treated plates, and the tensile direction is parallel to the rolling direction (Fig. 2). Both D-QP and P-QP were used to investigate the local microstructure evolution with the increasing tensile strain by EBSD (in PHI710). The electron backscatter diffraction data were acquired from the same region with the guidance of micro-Vickers indents on the detection surface (Fig. 2a). During electrolytic polishing, 


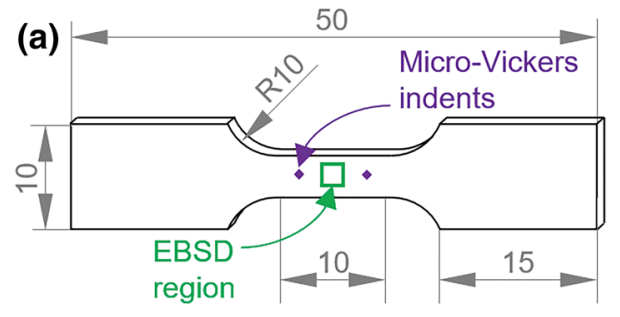

(b)

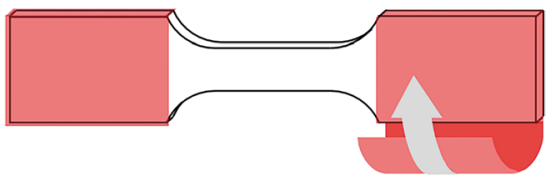

(c)

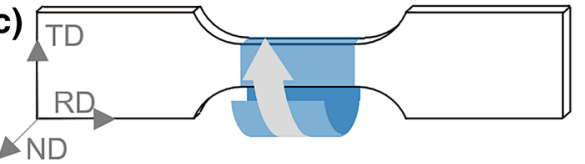

Fig. 2 Schematic diagrams of interrupted tensile plus quasi-situ EBSD samples: a specific dimensions (in $\mathrm{mm}$ ) of the specimen with the location of the micro-Vickers indents and the EBSD region; $\mathbf{b}$ schematic diagram of the specimen during electropolishing, red parts were wrapped with electrical tapes; $\mathbf{c}$ schematic diagram of the specimen during interrupted tensile tests, blue part was wrapped with electrostatic tape (ND: normal direction, TD: transverse direction, RD: rolling direction, the same below)

an electric tape was used to isolate the clamping end of the tensile specimen to achieve a better polishing effect on the inspection surface (Fig. 2b), and electrostatic tape protected the detection surface from pollution during interrupted tensile tests (Fig. 2c).

\section{Results and Discussion}

\subsection{Microstructures}

The initial microstructures of the D-QP and P-QP before the intercritical annealed Q\&P process are shown in Fig. 3a1 and $b 1$, respectively. The original microstructure of the $\mathrm{D}-\mathrm{QP}$ contains as-cold-rolled banded pearlite and ferrite, while that of the $\mathrm{P}-\mathrm{QP}$ is full martensite. The microstructure of the $\mathrm{D}-\mathrm{QP}$ before $\mathrm{Q} \& \mathrm{P}$ process is elongated along the rolling direction due to hot rolling and cold rolling. At the same time, the anisotropy in the microstructure of the P-QP before $\mathrm{Q} \& \mathrm{P}$ process is eliminated after a long period of complete austenitizing and quenching.

The microstructure of the steel is transformed into the mixture of ferrite, martensite and RA after the intercritical annealed Q\&P process. As shown in Fig. $3 a 2$ and b2, the microstructures of the D-QP and P-QP have different morphological characteristics. The former has the characteristics of equiaxed microstructure, while the latter is lamellar.

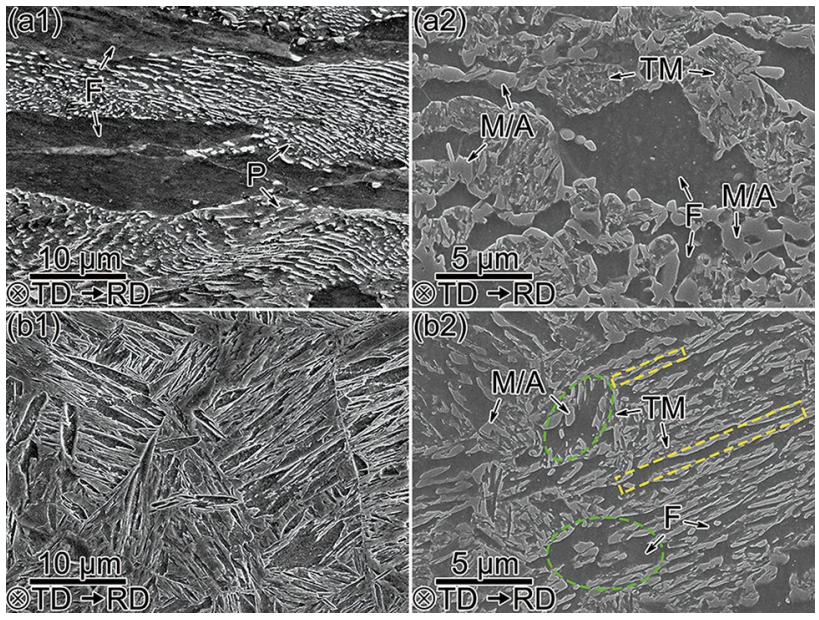

Fig. 3 SEM microstructures of the a D-QP b P-QP before $(-1)$ and after $(-2) \mathrm{Q} \& \mathrm{P}$ processes. The F, P, TM, and M/A represent ferrite, pearlite, tempered martensite, and martensite/austenite islands, respectively. The dotted boxes of ellipse (in green) and rectangle (in yellow) mark the typical areas of equiaxed ferrite and layered ferrite, respectively

Pre-quenching also changes the distribution of carbon in the samples. The cementite lamellae in pearlite are the carbon-rich part of the sample D-QP before Q\&P. Therefore, the interface between cementite and ferrite becomes the location of austenite nucleation during heating [24]. After pre-quenching, carbon is evenly distributed in martensitic matrix. When the $\mathrm{P}-\mathrm{QP}$ is heated to the intercritical region, austenite nucleates preferentially at the places with more defects, such as prior austenite grain boundary and martensite block boundary [25]. During heating, with the diffusion of carbon and the movement of austenite interface, original martensite decreases in volume fraction and transforms into ferrite with lower carbon content. After QP process, most of the ferrites remain the lamellar structure of martensitic block (yellow rectangles in Fig. 3b2), and few of them form equiaxed structure (green ellipses in Fig. 3b2), which exists at the grain boundary of prior austenite. Although the phase transition details of the D-QP and $\mathrm{P}-\mathrm{QP}$ are different in the intercritical annealed $\mathrm{Q} \& \mathrm{P}$ process, they all inherit the morphological characteristics of the initial microstructures.

Table 1 lists the phase volume fraction of the test steel, in which the volume fraction of ferrite is calculated from 10 SEM pictures, the volume fraction of RA is from XRD data, and then the rest is the volume fraction of martensite. The

Table 1 Phase volume fraction of the D-QP and P-QP (vol.\%)

\begin{tabular}{llll}
\hline & Retained austenite & Ferrite & Martensite \\
\hline D-QP & 15.5 & 35.5 & 49.0 \\
P-QP & 10.0 & 32.0 & 58.0
\end{tabular}



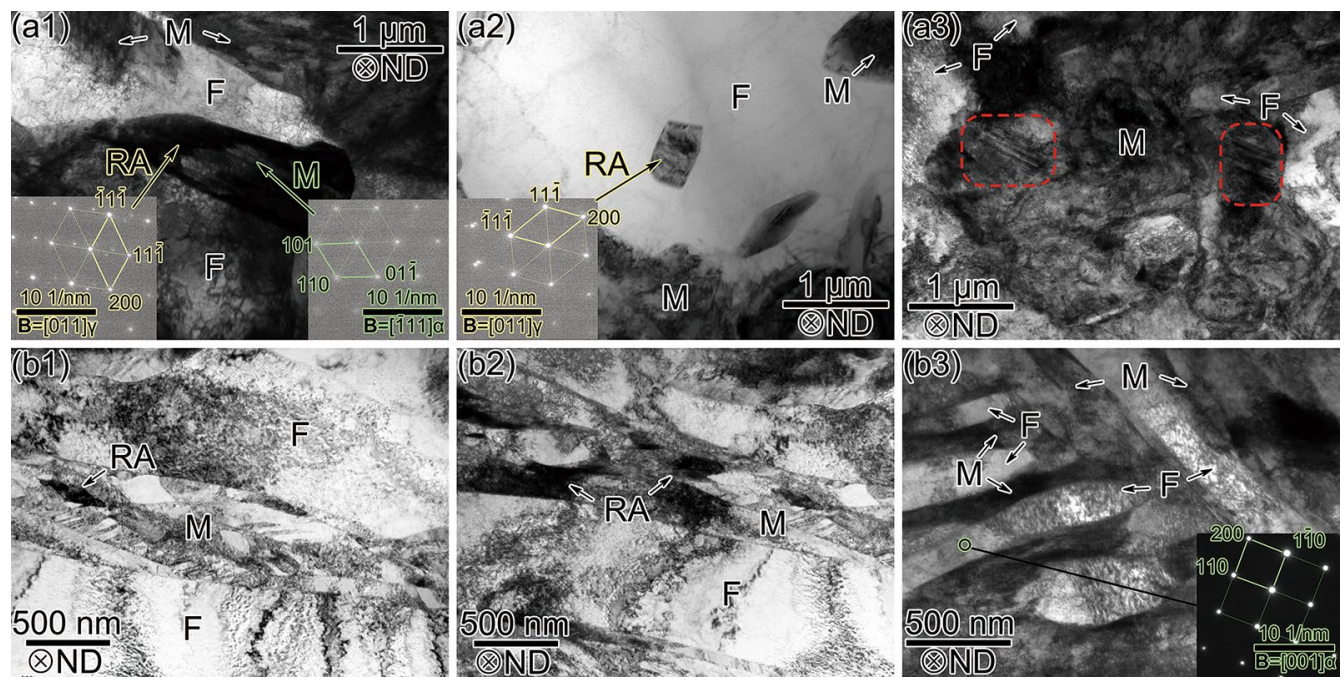

Fig. 4 TEM microstructures and diffraction pattern of the a D-QP b P-QP before $(-1,-2)$ and after $(-3)$ tensile tests. The F, M, and RA represent ferrite, martensite, and retained austenite, respectively. The dotted boxes of rounded rectangle (in red) mark the typical areas of twin

Table 2 Proportion and average size of RA with different morphologies

\begin{tabular}{|c|c|c|c|c|}
\hline & \multicolumn{2}{|c|}{ Equiaxed RA } & \multicolumn{2}{|l|}{ Film-like RA } \\
\hline & $\begin{array}{l}\text { Propor- } \\
\text { tion of } \\
\text { RA }\end{array}$ & Average size & Proportion of RA & Average size \\
\hline D-QP & $32 \%$ & $1.10 \mu \mathrm{m}$ & $68 \%$ & $0.12 \mu \mathrm{m}$ \\
\hline $\mathrm{P}-\mathrm{QP}$ & $0 \%$ & - & $100 \%$ & $0.10 \mu \mathrm{m}$ \\
\hline
\end{tabular}

difference in the RA volume fraction between the two specimens is due to the fact that the pre-quenching reduces $A_{\mathrm{c} 1}$ temperature of the sample P-QP [26].

In the sample D-QP, two types of RA, film-like and equiaxed RA, were observed by TEM. The equiaxed RA (Fig. 4a1 and a2) is thermally stable and fully retained during quenching, while film-like RA is embedded in martensite transformed during quenching. Only film-like RA (Fig. 4b1 and b2) was observed in the sample P-QP, which was distributed in lamellar martensite blocks. The proportion and average size of RA with different morphologies are listed in Table 2. After deformation (Fig. 4a3 and b3), twins and more dislocations appear in the sample D-QP and P-QP, but the microstructure of equiaxed or lamellar remains.

\subsection{Mechanical Properties}

The stress-strain curves and work hardening rate of the $\mathrm{D}-\mathrm{QP}$ and P-QP obtained by the uniaxial tensile test are illustrated in Fig. 5, and the mechanical properties are summarized in Table 3. It can be seen from the tensile test results that the P-QP has higher strength and elongation than the $\mathrm{D}-\mathrm{QP}$. The product of tensile strength and elongation of (a)

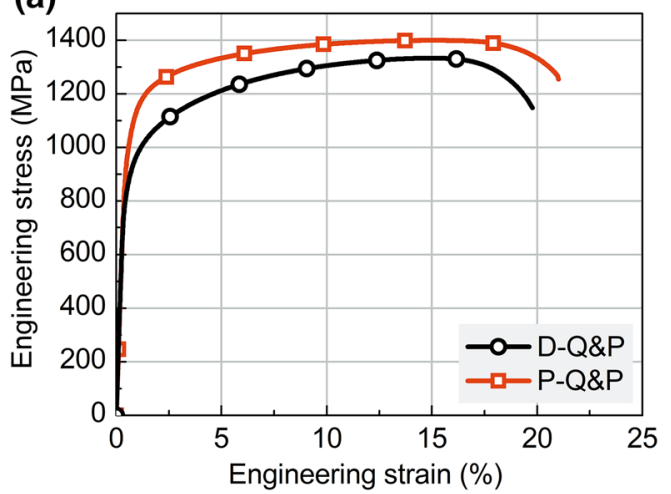

(b)

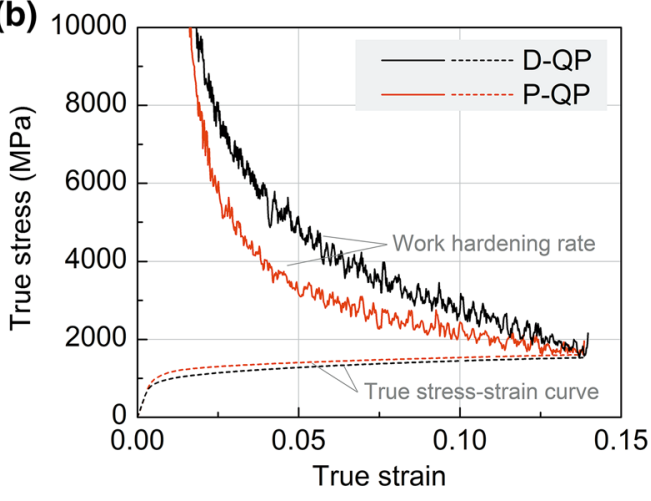

Fig. 5 Engineering stress-strain curves a, true stress-strain curves and work hardening rates b of the D-QP and P-QP 
Table 3 Mechanical properties of the D-QP and P-QP

\begin{tabular}{llllll}
\hline & Yield strength $(\mathrm{YS}, \mathrm{MPa})$ & Tensile strength $(\mathrm{TS}, \mathrm{MPa})$ & Total elongation $(\mathrm{TE}, \%)$ & YS/TS & TS $\times$ TE $(\mathrm{GPa} \%)$ \\
\hline D-QP & $858.29 \pm 21.25$ & $1333.29 \pm 9.35$ & $19.78 \pm 1.03$ & $0.6437 \pm 0.0114$ & $26.37 \pm 1.56$ \\
P-QP & $1024.82 \pm 28.41$ & $1401.09 \pm 12.13$ & $21.02 \pm 0.84$ & $0.7314 \pm 0.0139$ & $29.45 \pm 1.43$ \\
\hline
\end{tabular}

P-QP reaches $29 \mathrm{GPa} \%$, which has better performance in collision energy absorption.

Since continuous yielding is observed in each sample, the stress at a percentage non-proportional extension of $0.2 \%$ is used to characterize the yield strength (YS) of the specimens. After the intercritical annealed Q\&P process, a mixed microstructure of ferrite, martensite and austenite is formed. The homogenization of carbon caused by prequenching reduces $A_{\mathrm{c} 1}$ temperäwith sample D-QP, the lower ferrite volume fraction and the higher martensite volume fraction (Table 1) improve the yield strength of P-QP.

The volume fractions of RA in samples D-QP and P-QP are $15.5 \%$ and $10.0 \%$, respectively. The higher volume fraction of RA and TRIP effect in D-QP result in higher work hardening rate (Fig. 5b). In addition, the amount of RA has a direct impact on the yield ratio YS/TS [27]. For the D-QP, low yield ratios indicate a free mobility of the dislocations introduced by transformation of retained austenite into hard martensite during deformation.

\subsection{Microstructural Evolution During Interrupted Tensile}

To characterize the structural evolution of equiaxed and lamellar structures during plastic deformation of materials, especially the transformation behavior of RA, the interrupted tensile tests plus quasi-situ EBSD measurements were performed on the D-QP and P-QP. Figure 6 shows the engineering stress-displacement curves obtained from regular and quasi-situ tensile tests. According to the stress-displacement curves of the regular tensile tests, three stages of loading were applied on each interrupted tensile specimen. The displacements of $0 \mathrm{~mm}, 0.4 \mathrm{~mm}, 1.1 \mathrm{~mm}$ and $1.7 \mathrm{~mm}$ represent the elongations of the sample without deformation, after yielding, before ultimate tensile strength and at ultimate tensile strength, respectively. The elastic shrinkages of the specimens in the interrupted tensile tests are calculated using the data of the uniaxial tensile test.

Figure 7 shows the quasi-situ image quality (IQ) map of the D-QP and P-QP at different displacements. The green areas in this figure correspond to the fcc lattice (RA), while the darker color regions annotate the field of lower image quality value, which is caused by the grain boundary or dislocation. Although both ferrite and martensite are bcc lattice, they can be roughly distinguished by the image quality. Ferrite grains with less defects/ dislocation are lighter in the IQ map, while martensite grains are the opposite.

There are two distinct morphologies of austenite grains in the D-QP: the larger equiaxed grains (indicated by the arrows and arrowhead in Fig. 7a0) and the smaller film-like grains (in Fig. $7 \mathrm{a} 0$ without indicated by any arrows). The former can be divided into two types according to the different adjacent microstructures, namely the ferrite inclusion type (indicated by the arrows) and the martensite adjacent type (indicated by the arrowhead). The latter is distributed between martensitic laths or at the edge of martensitic block. Differently, there is only one morphology of austenite grains observed in the P-QP: the smaller film-like one. The distribution of carbon in the P-QP is relatively uniform when the austenite nucleation occurs from martensite in the intercritical annealing region, which causes a microstructure of lamellar austenite-ferrite after intercritical annealing. The
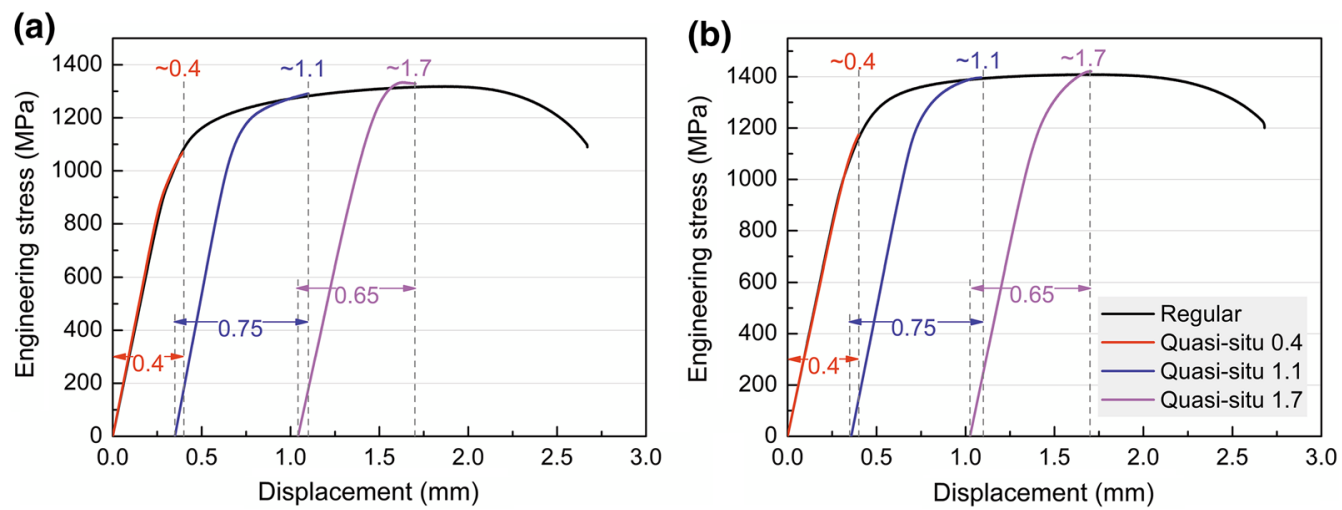

Fig. 6 Engineering stress-displacement curves obtained from regular and quasi-situ tensile tests of a D-QP b P-QP 


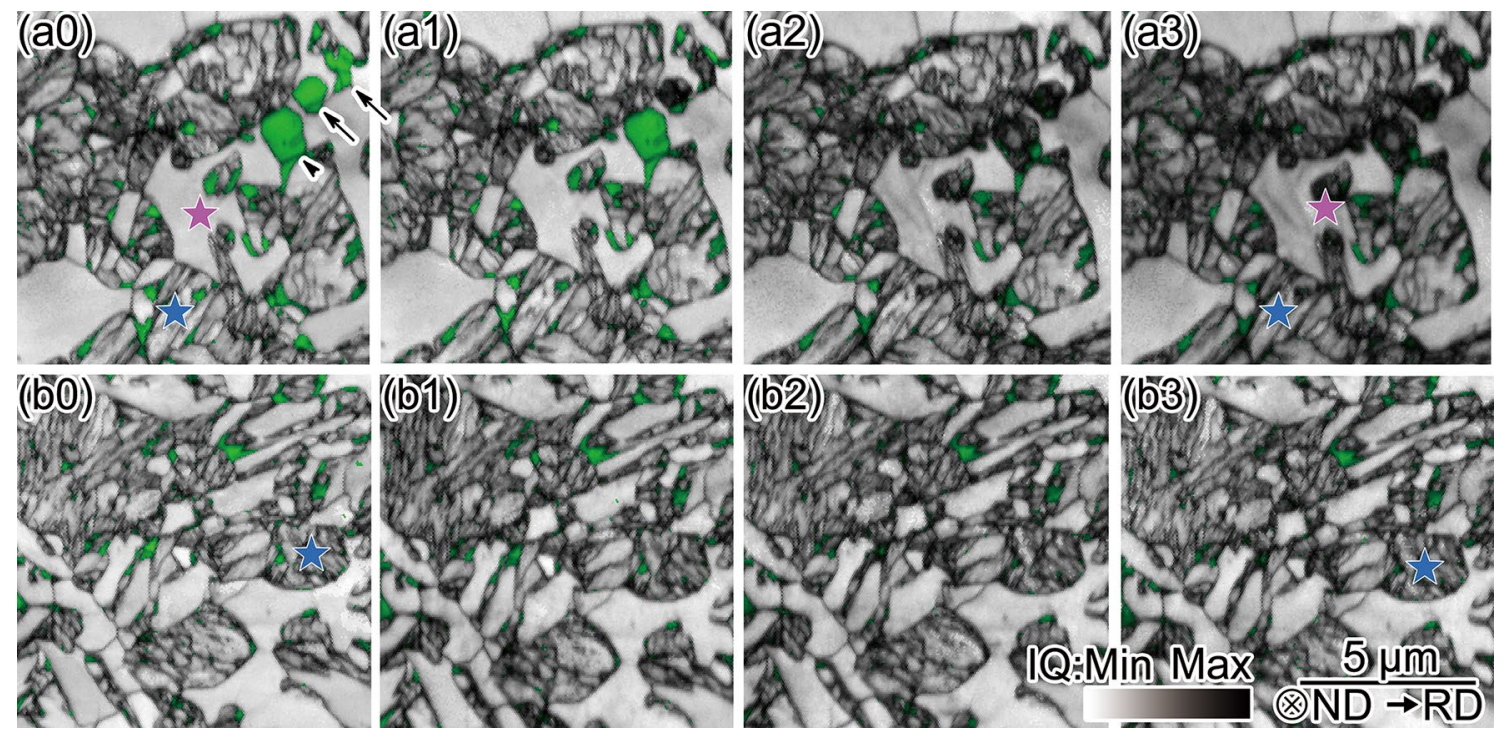

Fig. 7 Combined quasi-situ phase maps of RA and image quality (IQ) maps of a D-QP b P-QP at different displacements of (-0) 0 mm; (-1) $0.4 \mathrm{~mm} ;(-2) 1.1 \mathrm{~mm}$ and $(-3) 1.7 \mathrm{~mm}$. The green areas correspond to the fcc lattice. The arrows and arrowhead in Fig. $7 \mathrm{a} 0 \mathrm{indicate}$ equiaxed RA grains. The deformations of the grains at the five-pointed star positions are shown in Fig. 9
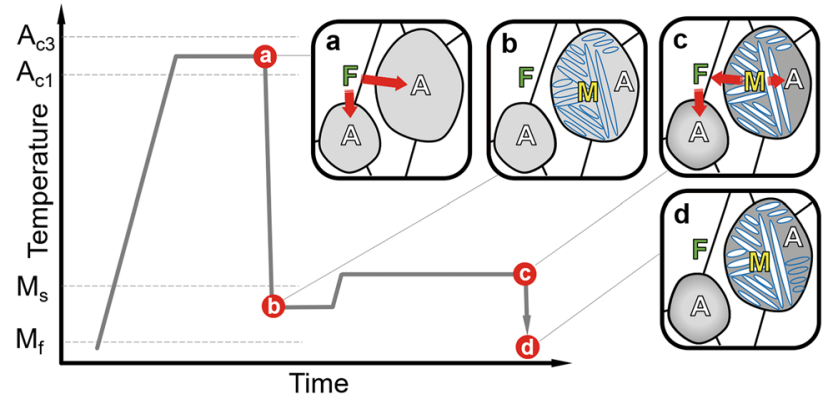

Fig. 8 Schematic diagram of the formation of RA in sample D-QP. The arrows corresponding the carbon diffusion direction

ferrite is divided by martensite block after quenching, while RA still follows the lamellar structure of martensite lath.

As shown in Fig. 7, the volume fraction of RA decreases upon displacement. In previous studies, the amount of martensite formation has been summarized as a function of strain [28] or/and stress [29]. Different types of RA also show a distinct order of transformation. Compared with the smaller lamellar austenite, the larger equiaxed one transforms into martensite earlier with the increase of displacement. At the same time, equiaxed RA wrapped by ferrite is the most unstable. These differences are due to the stability of RA (internal cause) and the structure around austenite grains (external causes). The explanation is as follows.

For the D-QP sample, the formation process of various types of RA is different, which directly leads to different carbon partitioning situations. Figure 8 shows the formation of RA in the D-QP. During the intercritical annealed
Q\&P treatment, there are two main carbon diffusion periods, which are intercritical annealing and isothermal partitioning treatments. The carbon in the ferrite diffuses into the austenite in the two-phase region (Fig. 8a), and the partially carbon-rich austenite transforms into martensite in the subsequent quenching stage (Fig. 8b). In the partitioning stage (Fig. 8c), the carbon in the martensite diffuses into the austenite. The carbon atoms must pass through the austenite at the edge of the martensitic block that may exist and the ferrite to reach the equiaxed austenite, leading to less carbon enrichment in the equiaxed RA grains than the film-like one. Devaraj et al. [30] visually characterized that the carbon content of lamellar RA inside the martensite is higher than the equiaxed type by using three-dimensional atom probe analysis. It is the difference in carbon content that makes several types of austenite have different stabilities. In the external factor, as shown in Fig. 9, during the strain process, the ferrite around the equiaxed RA has a larger deformation, while the deformation of the martensite region is smaller. In order to ensure the continuity of the microstructure, the equiaxed RA receives a larger microscopic displacement than the lamellar austenite inside the martensite. Similarly, the transformation of austenite wrapped by hard phase martensite is hindered.

Figure 10 shows the RA volume fraction of D-QP and $\mathrm{P}-\mathrm{QP}$ under different displacements. At the initial stage of strain, since the D-QP has two types of RA, i.e., equiaxed and film-like RA, the prior transformation of equiaxed RA makes the volume fraction of RA decrease significantly. However, the P-QP lacking equiaxed RA has a slight decrease. The point of $1.1 \mathrm{~mm}$ displacement separated the 


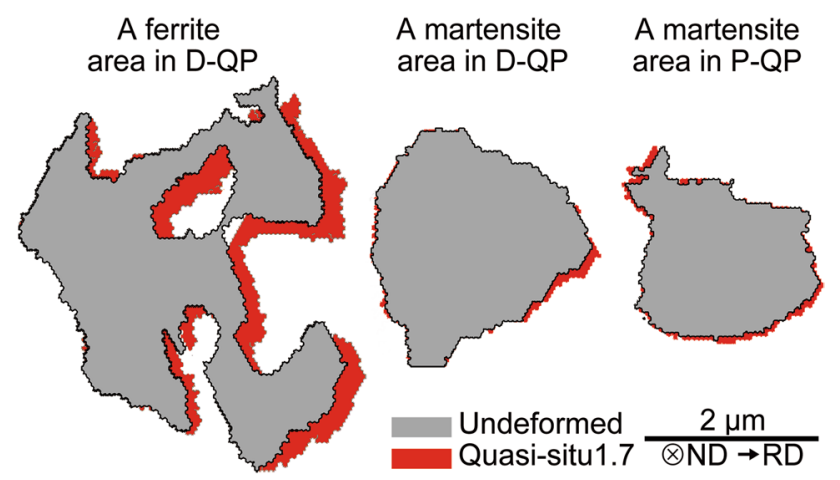

Fig. 9 Deformation of different phases after $1.7 \mathrm{~mm}$ displacement. The examined areas correspond to the positions shown by the fivepointed star in Fig. 7

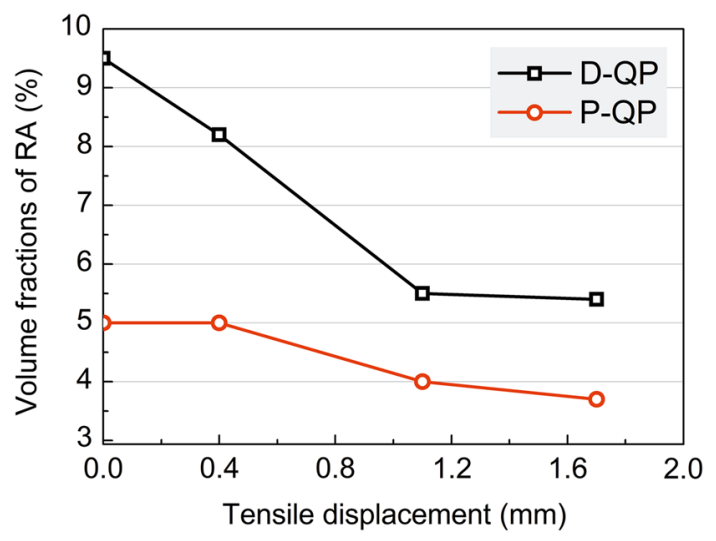

Fig. 10 RA volume fraction of D-QP and P-QP under different displacements
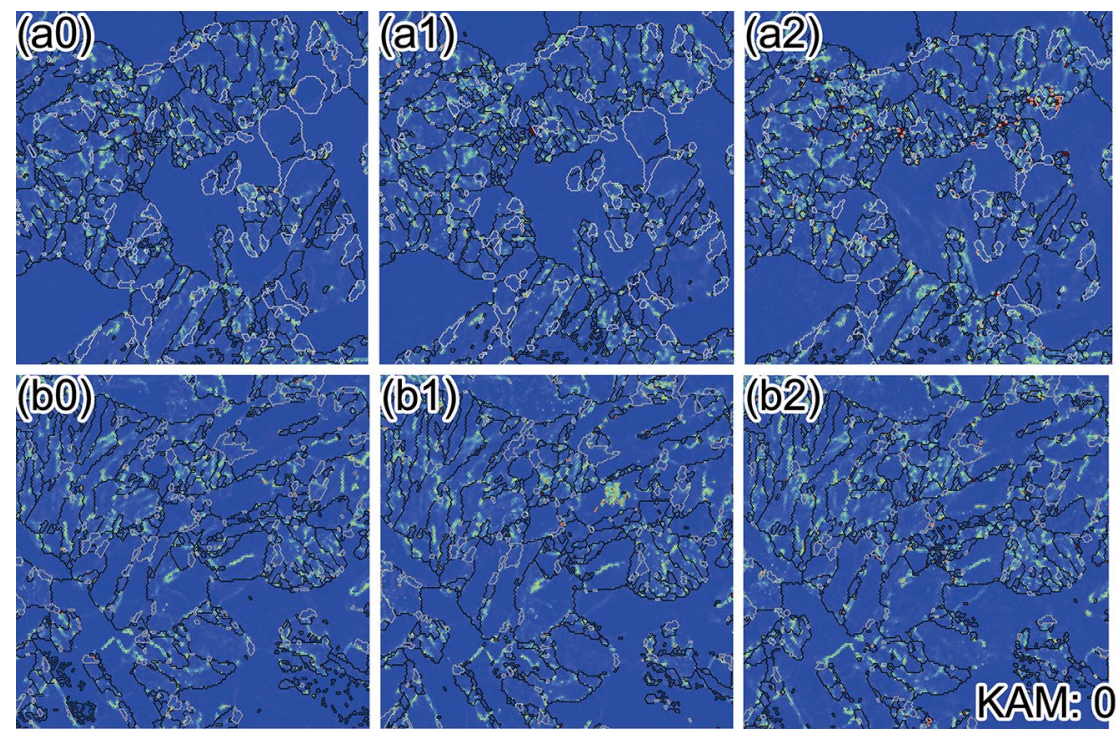

D-QP curve with different slopes. The decrease of slope indicates the completion of equiaxed RA transformation. However, the slope of the P-QP curve maintains stable, indicating that the austenite changes with displacement continuously.

Figure 11 shows the kernel average misorientation (KAM) changes of the D-QP and P-QP with displacement, and the corresponding KAM statistics are shown in Fig. 12. KAM probes pixel orientation difference and commonly indicates the geometrically necessary dislocation (GND), which maintains the continuity of the material under different internal strains. The difference in KAM value characterizes the gradient of the internal strain of the material.

As shown in Fig. 12, without deformation, the average KAM value of the $\mathrm{P}-\mathrm{QP}$ is higher than $\mathrm{D}-\mathrm{QP}$, which is mutually verified with the higher yield strength of sample P-QP. The average KAM value of the D-QP increases rapidly in the early stage of strain, which is attributed to the transformation of equiaxed austenite under small deformation. However, due to the uniform lamellar structure and the relatively single type of RA, the average KAM value of the P-QP growth rate remains stable.

Figure 13 shows the evolution of the KAM around $(\sim 120 \mathrm{~nm}) /$ of the austenite islands with different morphology (The corresponding KAM maps are shown in Figs. S2 and S3). Overall, the evolutions of the KAM around filmlike RA of D-QP and P-QP are similar, which are obviously different from the one of equiaxed RA in D-QP. In the initial stage of deformation, the KAM value of the film-like RA and its surrounding microstructure is high, and the high GND comes from the surrounding martensite with high

Fig. 11 Quasi-situ KAM maps of a D-QP b P-QP at different displacements of $(-0) 0 \mathrm{~mm} ;(-1) 0.4 \mathrm{~mm} ;(-2) 1.1 \mathrm{~mm}$ and $(-3) 1.7 \mathrm{~mm}$. The silver and black lines delineate the phase interface and the large angle grain boundary, respectively. The white dotted lines indicate the increase of KAM value of the local area 

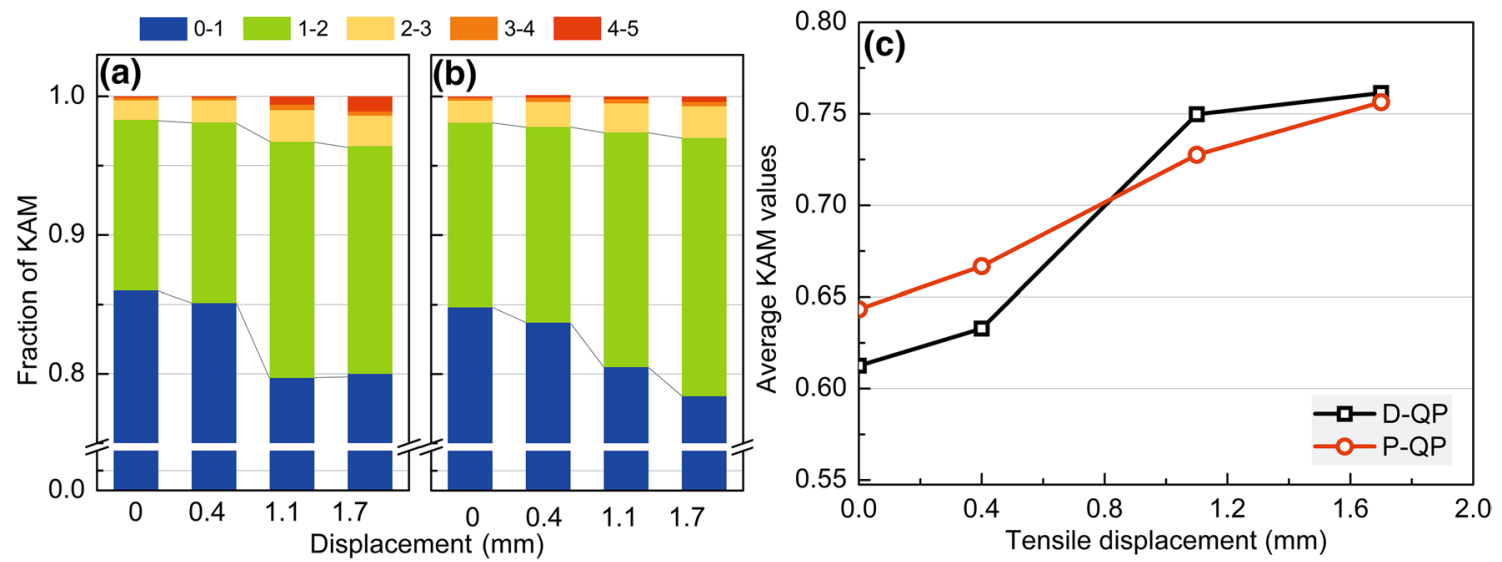

Fig. 12 Fraction of KAM of a D-QP b P-QP; $\mathbf{c}$ average KAM value of D-QP and P-QP under different displacements

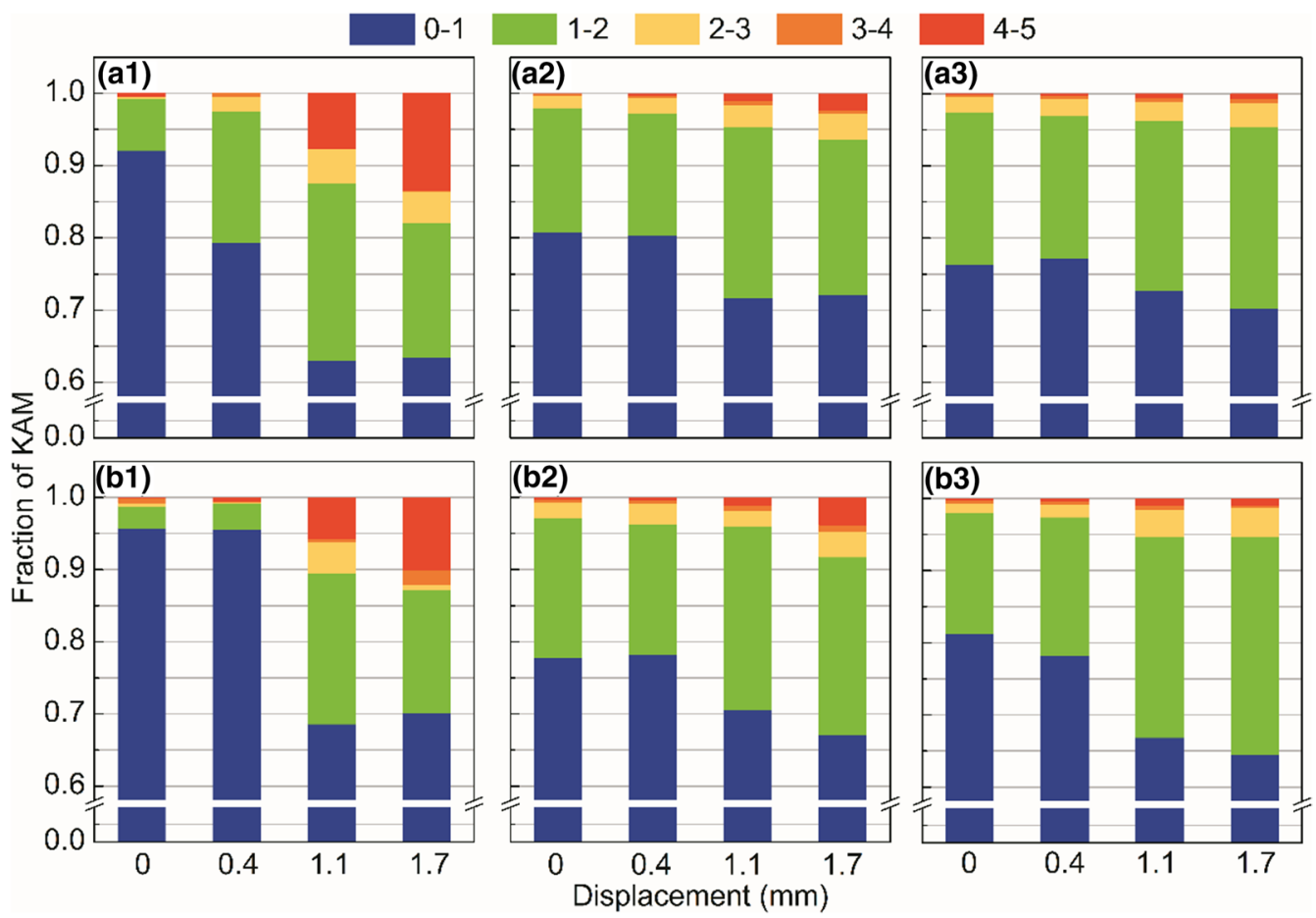

Fig. 13 Fraction of KAM a around $(\sim 120 \mathrm{~nm}) \mathbf{b}$ of $(-1)$ equiaxed RA in D-QP; $(-2)$ film-like RA in D-QP and $(-3)$ P-QP under different displacements

internal stress. As the strain progresses, the KAM around the equiaxed RA increases significantly, which is inseparable from the large amount of martensite transformation.

Previous studies [31-34] have shown that cracks tend to nucleate, expand, and coalesce along with the interface between matrix martensite and phase change martensite due to higher dislocation density. Around the sample D-QP yields, the transformation of equiaxed RA begins early, resulting in a significant increase in local dislocation density and stress concentration. Local hardening at the early stage of strain makes no contribution to slow down necking process but provides the location of crack initiation. However, the sample P-QP has a tiny amount of mechanically induced martensite at the initial stage of deformation due to the lack of equiaxed austenite. As shown in Fig. 11a3 and b3, compared with the sample P-QP, a nearly continuous network of high dislocation density regions (indicated by white dotted lines) caused by mechanically induced martensite was formed in the D-QP, which brings a lower fracture toughness [35]. The reasons discussed above will lead to a decrease in 
the ductility of the sample D-QP. Although the D-QP has a higher volume fraction of RA, the early transformation of equiaxed austenite and the formation of the brittle network, due to the equiaxed microstructure morphology, lead the transformation induced plasticity effect of RA less effective. The P-QP has uniform lamellar structure and the relatively film-like type of RA, which led to the steady increase in dislocation density upon straining, as well as better mechanical properties with the balance of strength and ductility.

\section{Conclusions}

The deformation behavior of equiaxed and lamellar microstructure was investigated between two low-carbon steels treated by intercritical annealed Q\&P process through interrupted tensile tests plus quasi-situ EBSD measurements, and the following conclusions are made:

1. Compared with the traditional equiaxed intercritical annealed Q\&P steel (D-QP), the pre-quenched steel (P-QP) with lamellar microstructure has higher yield strength, tensile strength, and elongation. The product of tensile strength and elongation of the latter reaches $29 \mathrm{GPa} \%$.

2. In D-QP, two types of RA, i.e., equiaxed and film-like $\mathrm{RA}$, were observed, while in P-QP, only the film-like RA was observed. The equiaxed RA included by ferrite has the lowest mechanical stability, followed by adjacent martensite. Significant martensitic transformation occurs in D-QP at the early stage of strain due to the existence of equiaxed RA.

3. The martensitic transformation of equiaxed RA at the initial stage of strain and the formation of brittle network with strain lead to the reduction of ductility of $\mathrm{D}-\mathrm{QP}$. The P-QP has uniform lamellar structure and the relatively film-like type of RA, which led to the steady increase of dislocation density upon straining, as well as better mechanical properties with the balance of strength and ductility.

Acknowledgements This work was supported by the National Key Research and Development Program of Thirteenth Five-year Plan Period (No. 2017YFB0304400); and the National Natural Science Foundation of China (No. 51574028).

\section{References}

[1] J.G. Speer, E. De Moor, A.J. Clarke, Mater. Sci. Technol. 31, 3 (2015)
[2] S.H. Sun, A.M. Zhao, R. Ding, X.G. Li, Acta Metall. Sin. -Engl. Lett. 31, 216 (2018)

[3] Z. Wang, M.X. Huang, Metall. Mater. Trans. A 50, 5650 (2019)

[4] B.B. He, M. Wang, L. Liu, M.X. Huang, Mater. Sci. Technol. 35, 2109 (2019)

[5] J.G. Speer, D.K. Matlock, B.C. De Cooman, J.G. Schroth, Acta Mater. 51, 2611 (2003)

[6] X. Wang, L. Liu, R.D. Liu, M.X. Huang, Metall. Mater. Trans. A 49, 1460 (2018)

[7] D.H. Kim, J.H. Kang, J.H. Ryu, S.J. Kim, Mater. Sci. Technol. 35, $2115(2019)$

[8] M.X. Huang, B.B. He, Mater. Sci. Technol. 34, 417 (2018)

[9] A.S. Nishikawa, G. Miyarnoto, T. Furuhara, A.P. Tschiptschin, H. Goldenstein, Acta Mater. 179, 1 (2019)

[10] D.D. Knijf, R. Petrov, C. Föjer, L.A. Kestens, Mater. Sci. Eng. A 615, 107 (2014)

[11] S.H. Sun, A.M. Zhao, Mater. Sci. Technol. 34, 347 (2018)

[12] A. Mark, M. Westphal, D. Boyd, J. McDermid, D. Embury, Can. Metall. Q. 48, 237 (2009)

[13] A. Zinsaz-Borujerdi, A. Zarei-Hanzaki, H.R. Abedi, M. KaramAbian, H. Ding, D. Han, N. Kheradmand, Mater. Sci. Eng. A 725, 341 (2018)

[14] L. Li, Z.L. Mi, Z. Wang, Y.G. Yang, Z.C. Yu, Mater. Res. Express 5, 066553 (2018)

[15] Y. Chong, G.Y. Deng, A.O. Yi, A. Shibata, N. Tsuji, J. Alloys Compd. 811, 152040 (2019)

[16] R. Ding, D. Tang, A. Zhao, Scr. Mater. 88, 21 (2014)

[17] R. Ding, Z. Dai, M. Huang, Z. Yang, C. Zhang, H. Chen, Acta Mater. 147, 59 (2018)

[18] X. Long, R. Zhang, F. Zhang, G. Du, X. Zhao, Mater. Sci. Eng. A 760, 158 (2019)

[19] J. Zhao, F. Zhang, Mater. Sci. Eng. A 711, 138637 (2020)

[20] O. Matsumura, Y.S. Sakuma, H.S. TakeChi, Trans. Iron Steel Inst. Jpn. 27, 570 (1987)

[21] J.G. Speer, D.K. Matlock, B.C. DeCooman, J.G. Schroth, Scr. Mater. 52, 83 (2005)

[22] A.J. Clarke, J.G. Speer, M.K. Miller, R.E. Hackenberg, D.V. Edmonds, D.K. Matlock, F.C. Rizzoe, K.D. Clarkea, E. De Moorf, Acta Mater. 56, 16 (2008)

[23] J. Jung, S.J. Lee, S. Kim, B.C.D. Cooman, Steel Res. Int. 82, 857 (2011)

[24] D.V. Shtansky, K. Nakai, Y. Ohmori, Acta Mater. 47, 2619 (1999)

[25] D. De Knijf, C. Föjer, L.A.I. Kestens, R. Petrov, Mater. Sci. Eng. A 638, 219 (2015)

[26] S.H. Sun, A.M. Zhao, R. Ding, X.G. Li, Acta Metall. Sin. -Engl. Lett. 31, 216 (2017)

[27] P. Huyghe, L. Malet, M. Caruso, C. Georges, S. Godet, Mater. Sci. Eng. A 701, 254 (2017)

[28] D.C. Ludwigson, J.A. Berger, J. Iron Steel Inst. 207, 63 (1969)

[29] I. Tamura, Met. Sci. 16, 245 (1982)

[30] A. Devaraj, Z. Xu, F. Abu-Farha, X. Sun, L.G. Hector, JOM 70, $1752(2018)$

[31] S. Zhang, E. Fan, J. Wan, J. Liu, Y. Huang, X. Li, Corros. Sci. 139, $83(2018)$

[32] J. Samei, Y. Salib, M. Amirmaleki, D.S. Wilkinson, Scr. Mater. 173, 86 (2019)

[33] Z. Xiong, P.J. Jacques, A. Perlade, T. Pardoen, Metall. Mater. Trans. A 50, 3502 (2019)

[34] J. Hidalgo, C. Celada-Casero, M.J. Santofimia, Mater. Sci. Eng. A 754, 766 (2019)

[35] P. Jacques, Q. Furnemont, T. Pardoen, F. Delannay, Acta Mater. 49, 139 (2001) 